\title{
Issues in the specification of regret-only choice models: a rejoinder to Chorus and Van Cranenburgh
}

\author{
Soora Rasouli ${ }^{1} \cdot$ Harry Timmermans ${ }^{1}$
}

Published online: 25 October 2016

(C) The Author(s) 2016. This article is published with open access at Springerlink.com

Before starting the debate, we would like to state explicitly that we share Chorus and Cranenburgh's $(\mathrm{C} \& \mathrm{C})$ concerns about prevailing confusion about regret-based models and their fascination of exploring the value of context-dependent choice models in transportation research and beyond. Although the number of applications of random regret models has gradually increased over the last decade, still there are many nonbelievers as evidenced by critical, skeptical questions at conferences, questionable paper reviews and the contents of informal discussions. First, some peers have questioned their theoretical foundation, as they are not based on classic axiomatic theorems of rational decision-making. Second, the relevance of random regret models has been downplayed as empirical studies have shown that even if these models perform better than random utility models, their performance is only marginally better. Therefore, it has been argued their further development is not very worthwhile. Thirdly, compromise effects may have been observed in some marketing studies, doubts have been raised about their importance in transportation. Finally, we have often heard that because choice probabilities in both the MNL and random regret models are based on utility/ regret differences, the models are fundamentally the same; although most MNL models assume a linear-additive utility function, there is no reason not to use non-linear (reference-based) functions, fading out their difference. These skeptics argue we do not need the concept of regret; the concept of utility is sufficient to formulate more flexible choice models. These reactions suggest a lot of confusion and lack of understanding of regret models. We have no desire to further contribute to this situation and therefore like to take this opportunity to further explain the considerations that led to our 2016 paper (Rasouli and Timmermans 2016) and articulate our opinions in response to C\&C's comments.

To start, it may be fair to acknowledge that part of the confusion may have been caused by the gradual evolution of regret-based models. Over the years, small incremental changes

Soora Rasouli

s.rasouli@tue.nl

1 Urban Planning Group, Eindhoven University of Technology, Eindhoven, The Netherlands 
have been suggested and new insights and interpretations have been added. It is therefore critically important that readers are aware of the state of the art at the time of the design of the study reported in our paper and the writing of the results. This is particularly the case because $\mathrm{C} \& \mathrm{C}$ argue that we "overlooked past work on the topic". In response, we did not overlook past work; the paper is based on the state of the art when the paper was written. When we designed the study, the only available publications on regret-based models in travel behavior research were the original Chorus et al. (2008) paper on the random regret model (RRM2008) and the paper on Chorus' (2010) specification (RRM2010) plus a series of article, listed in our paper, comparing the empirical performance of the latter model against the MNL model. ${ }^{1}$

C\&C use the opportunity to promote and summarize their latest (2014-2015) publications. For those readers, not familiar with their recent publications, it gives an excellent overview of developments in regret-rejoice models after we conducted our study. It is tempting to express our (full) assessment of these new models, but we decided not to as it would not be fair to the authors, who would not have the opportunity to respond to the discussion. Rather, we prefer to rely on submitted papers and work in progress for a detailed discussion of some specific issues. Jang et al. (2016) is the first of a larger series of papers in which we aim at improving and extending regret and regret-rejoice models, both under certainty and uncertainty, often based on a critical assessment of a particular assumption or property of existing models. At the same time, we realize this may be disappointing for readers as most of $\mathrm{C} \& \mathrm{C}$ arguments are based on their latest work and latest insights.

Therefore, we limit this rejoinder to the comment on "erroneous theoretical claims" and include their more recent work to the extent we think it helps in avoiding confusion. Critical for the positioning of our paper is to realize that, in contrast to seminal work on regret models, the 2008 specification is a regret-only model. Regret for each attribute was assumed to be proportional to the difference between the attribute levels of the considered/chosen alternative and the attribute level of the BEST foregone choice alternative. In addition, overall regret, defined as the sum of attribute level regret, was also defined against the best non-chosen choice alternative. In equation:

$$
R_{i}=\max _{i^{\prime}} \sum_{k}\left(\max \left(0, \beta_{k}\left(x_{i^{\prime} k}-x_{i k}\right)\right)\right) .
$$

It should be noted that the formulation of this regret-only model does not imply we believe regret-only models are necessarily superior. Rather, the regret-only models serve as an interesting benchmark to differentiate the specification from seminal regret theory

\footnotetext{
1 Our first discussion about regret models took place in the Spring of 2013. We needed to develop research proposals for new PhD students, and decided to pick up again the topic as a line of research. Our discussion of the state of the art led to the small-scale project (RT2016) as a modest start and side-project, and the formulation of a series of much more ambitious $\mathrm{PhD}$ research projects. The data were collected on July 15 , 2013, and the first draft of the paper was submitted in January 2014. C\&C's new insights were published much later and because we did not belong to the reviewers of their work, we were not aware of it. It took a long time until our Transportation paper was published, primarily because we were slow in submitting the final revised version due to deadlines of large scale projects. Then, the paper got lost in the production process, which we only realized when another of our papers on regret models (Jang et al. 2016), which was accepted much latter, was published on line sooner. By the time we became aware of C\&C latest work, the paper was already accepted.
} 
introduced in decision sciences ${ }^{2}$ and regret-based decision heuristics examined as rules of bounded rational behavior (see e.g. Rasouli and Timmermans 2015). We believe it is valuable to explore the theoretical properties of regret-only models and assess their empirical performance against utility-maximizing and regret-rejoice models. The main contribution of the 2008 study is that it showed the potential of random regret models. Still, several technical, econometric and behavioral aspects of the model could and should be systematically investigated and possibly improved.

In his 2010 paper (RRM2010), Chorus addressed one of these issues: the discontinuous regret function may cause problems in the estimation of the regret function. As an alternative, he suggested the following specification.

$$
R_{i}=\sum_{i^{\prime} \neq i \in c} \sum_{k=1}^{K} \operatorname{In}\left(1+\exp \left(\beta_{k}\left(x_{i^{\prime} k}-x_{i k}\right)\right)\right) .
$$

Note that if we leave out the constant, the two regret functions are identical. If attribute differences are large, the effect of the constant is negligible, but differences between the two specifications increase if attribute differences are small. When the attribute values of the considered and the best alternative are the same, regret is positive while we argue logically it should be equal to zero because the considered/chosen alternative is the best and therefore there cannot be any regret. This is also clearly shown in Chorus' RRM2010 Fig. $1{ }^{3}$ We argued this is theoretically weak. In turn, C\&C claimed that our argument is incorrect and concluded that subtracting $\ln (2)$ from the value of the attribute regret function will solve the problem that RRM2010 gives non-zero regret even if the chosen alternative outperforms the other(s). ${ }^{4}$

Their wording is as if this "solution" is something we did not realize. This is surprising and misleading because in our paper, we presented an equation that captures the difference between the original regret function and the RRM2010 approximation. It shows that under the same attribute values the difference between the two specifications is $\ln (2)$ : exactly the "solution" they suggest. It is even more interesting to read C\&C's diagnostic conclusion that we do not understand the basics of choice modeling and regret theory. Upon selfreflection, we like to believe that we know the basics of discrete choice and regret theory at least well enough to understand their argument and to argue it is not a satisfying "solution" as it would enhance the confusion in the field that we would like to avoid or temper: we learned that many peers do think that because choice probabilities under utility-maximization only depend on utility differences, the properties and assumptions of the MNL model can be fully generalized to regret models. Mathematically, this is correct; parameter estimates only depend on the differences; goodness of fit and predicted choice probabilities

\footnotetext{
${ }^{2}$ Seminal regret theory of decision under uncertainty is based on the different notion that people not only take into account the utility that they derive from their choices but also what they might have gotten had they chosen differently. Regret indicates that an individual could have done better by choosing other alternatives. Seminal models thus include a utility function and a function capturing the impact of regret. The converse experience has been called rejoicing. The principle of regret aversion predicts violations of transitivity that are not (easily) accommodated by expected utility theory and other non-expected utility theories, such as prospect theory.

3 The comments led us to re-read the original RRM2010 paper. Maybe the intention was not as we assumed to find a close approximation of the 2008 specification. Figure 1 suggests the new function was meant to be a smooth approximation. Even if that interpretation would be incorrect, our comparison still holds.

4 We note this suggestion was not made in the original RRM2010 paper. Their discussion appeared in their 2014 and 2015 papers.
} 
are not affected; no discussion and no confusion. ${ }^{5}$ Our point is that as behavioral modelers we need to superimpose additional requirements. The concept of utility is a continuous concept, usually measured at an interval scale. The concept of regret is not. Logically and operationally it is only defined in the positive domain. Allowing for violations would fuel the notion that the concepts of utility and regret are behaviorally very similar. We do not question that choice probabilities are a function of regret differences. However, we do prefer model specifications that have a direct behavioral interpretation and that do not depend on some ad hoc correction. It is a non-issue in utility models as the concept of utility goes from minus to plus infinity. In contrast, however, regret is only defined for the positive domain and we prefer arguments in the regret function that have this property. We realize that one can take a different stance on this issue. We simply prefer to avoid any motivation based on classic discrete choice models, as C\&C do, to correct matters in an ad hoc fashion. We particularly prefer this because we can easily assume strictly positive regret functions for regret-only models.

More importantly, subtracting $\ln (2)$ is NOT a solution. Indeed, as implied in the equation we presented, regret in RT2016 becomes zero for zero attribute differences. However, it will shift the complete regret function. Consequently, regret may become negative when the attribute of the considered alternative outperforms the attribute of the best/another choice alternative. This violates the very notion of regret. It also is problematic because our discussion concerns regret-only models. Recall the reason for the new regret specification was to obtain a smooth approximation of the original function. Chorus (2010) states: "Second, the (2008) model specification's likelihood function is nonsmooth, which creates difficulties with respect to the derivation of marginal effects and elasticities, and triggers a need for customized optimization routines to successfully estimate the model. This means that model estimation relies on handwritten code, which in turn hampers the model's general applicability, especially among practitioners". This is a practical argument. The underlying problem could be easily solved in other ways (e.g., dedicated software (stand alone or included in general software such the 2010 model now is), smaller constant, other function), avoiding a regret function that in C\&C own words seems (at first glance) counterintuitive. ${ }^{6}$

By focusing on this minor issue, $\mathrm{C} \& \mathrm{C}$ seem to take away the focus from the more important point that the two regret functions differ when attribute differences are small. Subtraction of $\ln (2)$ makes the value of regret based on the max operator and logarithmic function equal only when the two alternatives have exactly the same values for the corresponding attribute. Equalizing the two regret specifications is not as straightforward as they claim. ${ }^{7}$ There remains a difference between the two specifications for small attribute differences, which led to the dedicated design of our stated choice experiment.

\footnotetext{
5 The fact that parameters estimates only depend on differences does not imply we should not be concerned with the valid representation of concepts.

${ }^{6}$ We would not have any problems with the argument that the linear regret function may not be a good representation and that there would be good behavioral reasons to examine non-linear functions. In fact, this is part of our research agenda, although we examine different non-linear functions that are consistent with our position expressed in this rejoinder.

7 Only in later publications, the concepts of rejoice and risk avoidance appeared to justify the meaning of the shift in the regret diagram by subtraction of $\ln (2)$. The concepts of rejoice does not appear in Chorus (2010). In our opinion, subtracting $\ln (2)$ to make the formulation consistent with the very basic notion of regret, while simultaneously trying to attach a meaning to this shift does not seem very rigorous. Moreover, it turns a regret-only into a regret-rejoice model.
} 
Another, and maybe more important difference between the two models, is whether people experience or consider regret against the best choice alternative only or against all better choice alternatives. The RRM2008 paper provides arguments in favor of the best only model. Stimulated by one of our reviewers, our 2016 paper also emphasizes arguments in favor of best only models. In contrast, C\&C discussed limitations of best only models, and we have also expressed theoretical reasons why regret-rejoice models may be more appealing. Anyhow, the RRM2010 paper suggests regret is based on all pairwise comparisons. ${ }^{8}$ As said, stimulated by one of the reviewers, we emphasized in RT2016 why regret may be viewed against the best foregone alternative only. Ultimately, however, we said whether regret is defined against the best only alternative or against all other alternatives is an empirical issue. We can imagine that if a person uses the first petrol station he encounters and then finds out that all next stations along his route have cheaper gas, his regret will be higher than in the case he finds out all other stations but one (the absolutely cheapest) were more expensive. On the other hand, we doubt regret will significantly increase if a traveller knows that sometimes his habitual route from work to home becomes extremely congested and he would have been better off by choosing one of approximately equivalent, strongly overlapping alternative routes. More importantly, because we view behavioral models as mathematical representations of assumed behavioral processes, we have difficulty assuming that individuals cognitively assess the amount of regret in large choice sets, such as housing, route choice and the like by systematically comparing all choice alternatives. Thus, we feel it is important to formulate a model of the mental representation of the choice problem that will predict the number of choice alternatives that will be included in the comparison. ${ }^{9}$ Our experiment therefore allowed the comparison of the best only versus all comparisons specifications, admittedly in a still limited choice problem context.

The argument of the number of case studies is too far-fledged in our opinion. We would never claim the general superiority of one model against another on the basis of a single data set. Also, we do not fundamentally prefer the 2008 model to the 2010 model: we just compared their performance. Our criticism only concerns the specification of the regret function that has counterintuitive properties for regret-only models that can be easily avoided. We again want to emphasize that the focus of our comparison concerned the relative performance of these two model specifications for small attribute differences, because the two model specifications are approximately identical for larger differences.

If C\&C's argument is meant to suggest that no one should submit papers for publication until a model has been successfully applied to many data sets, we fundamentally disagree. We would not have seen the original regret model until much later. Waiting until a particular model has shown to outperform another for a large number of data sets does not seem very productive. We should stimulate that if a new model seems to have potential (there is evidence it outperforms a well-established model), it will be accepted in journals. This increases the chances that peers will estimate the model on new data and suggest improvements. That is called progress and avoids inertia. We do expect that the performance of a particular choice model will depend on the choice problem and choice context. It is more interesting to examine whether it is possible to find conditions or type of problems under which a particular model tends to outperform others and to identify the reasons why it does not. This research may enrich the specification of the context in which

\footnotetext{
${ }^{8}$ It makes this version of the random regret model has much in common with the relative utility model, suggested by Zhang, et al. in their 2004 Transportation Research B paper.

9 In fact, the relative utility model suggested in Zhang et al. (2004) allows for this.
} 
a particular choice mechanism seems dominant. We need a spectrum of principles and corresponding models and can only hope that we find some degree of regularity between choice mechanisms and choice context. Rather than taking a defensive stance, we would like to stimulate our research community to present their findings (positive and negative) of applications of existing model specifications at conferences and workshops and that reviewers be more liberal with respect to acceptance of any new specifications in the journals. Having said that, in the meantime, we did compare the two model specifications using new, both revealed and stated preference, data sets. The conclusions in our 2016 paper were confirmed for these other data sets (see e.g. Jang et al. 2016). It shifts the support ratio slightly, but again this is less important than trying to understand why a particular model outperformed another.

In summary, filtering out and ignoring the unjustified, unnecessarily suggestive and unwarranted comments, we arrive at the following contentions.

1. To avoid any confusion, we re-emphasize that our RT2016 paper only considers the regret-only choice models, published in 2008 and 2010, simply because the other model specifications were published after our paper was accepted. C\&C more recent work is not about regret-only but about regret-rejoice models. These models indeed are much richer in that they allow estimating the effects of regret and rejoice, but our basic concerns of direct interpretability equally apply to these models, except for some asymptotic cases.

2. Because we highly value the methodological principle that the measurement and operationalization of behavioral constructs should satisfy definitional, logical and datatheoretical properties of the constructs, we prefer the specification of the attribute level regret function in RRM2008 to RRM2010 for its direct interpretation and avoidance of what C\&C (2015, p. 93) later called counterintuitive associations of the RRM2010 specification.

3. The suggested solution to subtract $\ln (2)$ from the regret function is not a solution if the representation concerns a regret-only model and RRM2010 is meant to be a smooth approximation of RRM2008.

4. If the aim of RRM2010 was to introduce a non-linear attribute level regret function, other directly interpretable functions for regret-only models can be easily formulated, and hence would have our preference. Such functions can still have all the properties that $\mathrm{C} \& \mathrm{C}$ find so desirable.

5. The issue whether a best-only or a pairwise comparison regret model best represents choice processes is an empirical issue, but the pairwise comparison version (RRM2010) is behaviorally difficult to defend for choice problems that involve many options if it is accepted that under those conditions people tend to use simplifying strategies to reduce the cognitive burden of comparing alternatives, and that the mental effort involved is a function of the discriminatory power of the attribute levels.

6. We re-iterate, evidenced in our publications, that we do not take any extreme stance as to the a priori superiority of any behavioral paradigm or model specification. In fact, we feel such a stance is counter-productive for academic progress.

We wish to emphasize, although this should be self-evident, that our reflections are not meant in any way to question the general quality and important contributions of Chorus and his co-authors. In fact, we think their work has given new impetus to behavioral choice modeling in transportation that started to show signs of satiation, consolidation of old positions and marginalization of progress. 
In that context, we would like to argue that the issues debated here are minor compared to the issues mentioned in our introduction. A larger research agenda continues to further critically question and examine the behavioral and econometric assumptions of current regret-only and regret/rejoice choice models, including C\&C 2014 and 2015 generalized versions and our own work, explores the performance of alternative formulations, and expands these models into more complicated classes of choice problems. We expect to systematically investigate these issues with our $\mathrm{PhD}$ students and in joint publications with Chorus and van Cranenburgh in future work.

Open Access This article is distributed under the terms of the Creative Commons Attribution 4.0 International License (http://creativecommons.org/licenses/by/4.0/), which permits unrestricted use, distribution, and reproduction in any medium, provided you give appropriate credit to the original author(s) and the source, provide a link to the Creative Commons license, and indicate if changes were made.

\section{References}

Chorus, C.G.: A new model of random regret minimization. Eur. J. Transp. Infrastruct. Res. 10, 181-196 (2010)

Chorus, C.G., Arentze, T.A., Timmermans, H.J.P.: A random regret-minimization model of travel choice. Transp. Res. B 42, 1-18 (2008)

Jang, S., Rasouli, S., Timmermans, H.J.P.: Incorporating psycho-physical mapping into random regret choice models: model specifications and empirical performance assessments. Transportation 9, 1-22 (2016)

Rasouli, S., Timmermans, H.J.P.: Models of bounded rationality under certainty. In: Rasouli, S., Timmermans, H.J.P. (eds.) Bounded rational choice behavior: applications in transport. Emerald Publishers, Chennai (2015)

Rasouli, S., Timmermans, H.J.P.: Specification of regret-based models of choice behavior: Formal analyses and experimental design based evidence, Transportation, 43, accepted for publication (2016)

Zhang, J., Timmermans, H.J.P., Borgers, A.W.J., Wang, D.: Modeling traveler choice behavior using the concepts of relative utility and relative interest. Transportation Research Part B: Methodological 38(3), 215-234 (2004)

Soora Rasouli is Associate Professor of urban planning group, at TU Eindhoven. Her main research interests concern the development of behavioral choice models, the advancement of comprehensive activitybased models and uncertainty in complex systems.

Harry Timmermans is Head of the Urban Planning Group, Eindhoven University of Technology. His main research interest concerns the analysis and modeling of decision making processes, differentiating between choice contexts and application domains. 